Special Issue of the 8th International Advances in Applied Physics and Materials Science Congress (APMAS 2018)

\title{
Natural Nanohydroxyapatite Synthesis via Ultrasonication from Donax Trunculus Bivalve Sea Shells and Production of its Electrospun Nanobiocomposite
}

\author{
Y.M. SAHIN ${ }^{a, b, *}$ \\ ${ }^{a}$ Istanbul Arel University, ArelPOTKAM (Polymer Technologies and Composite Application and Research Center), \\ Buyukcekmece, 34349, Istanbul, Turkey \\ ${ }^{b}$ Istanbul Arel University Department of Biomedical Engineering, 34349, Istanbul, Turkey
}

\begin{abstract}
In the present study, hydroxyapatite (HA) and tricalcium phosphate (TCP) bioceramics were prepared via a practical, ultrasonic conversion method from Donax trunculus seashells. These seashells are one of the most common bivalve molluscs of the Mediterranean Sea and can be used as a natural, stable raw material for bioceramic production. Ultrasonication, a powerful method for nano-sized ceramic production, was chosen to synthesize different ceramic phases easily. Raw shells are consisted of calcite and aragonite structures. To synthesize HA and TCP bioceramic materials, first the calcium oxide content of the shells were identified via Differential Thermal Analysis (DTA) and then a calculated amount of phosphoric acid was added drop by drop to obtain the exact stoichiometry. After synthesis, the resultant bioceramics were sintered at $800-850{ }^{\circ} \mathrm{C}$ for $\mathrm{HA}$ and $400-450{ }^{\circ} \mathrm{C}$ for $\mathrm{TCP}$ phases. For bioceramic phases X-ray Diffraction (XRD), Fourier Transform Infrared Spectrometer (FTIR), Field Emission Gun Scanning Electron Microscope (FEGSEM) studies were perform. On the other hand, electrospinning method was used to prepare nanobiocomposites from biocompatible polymeric material as the matrix and the obtained natural bioceramics as reinforcer of the composite system. Three different compositions were used and optimum electrospinning conditions were adjusted to prepare these electrospun structures. Biocomposites were evaluated in terms of structure, mechanic, morphology and biology. The effect of bioceramic content was also discussed. It is revealed that the obtained electrospun nanobiocomposites are good candidates for various tissue engineering purposes due to their enhanced biological and mechanical properties.
\end{abstract}

DOI: 10.12693/APhysPolA.135.1093

PACS/topics: marine sourced bioceramics; ultrasonic conversion; electrospun biocomposites; tissue engineering

\section{Introduction}

Biocomposites have recently been the focus of much research to enhance biological and mechanical properties of the biomaterial. An encouraging challenge will be to built up nanofiber structures that enhance survival and uniform distribution of cells resulting superior properties. These systems should be stiff enough to ease handling, while elastic enough to limit damage to newly occured tissues [1]. Thus a composite material incoorporated bioceramic and polymeric phases are presented purposefully in this study. In view of the variety of roles played by bioceramics in different tissues, researches have focused on developing novel biomaterials to mimic the bone structure. Bone is a composite structure composed of HA crystalls that dipersed througout an biocompatible polymeric matrics [2]. In the proposed study, in order to mimic bone structure, beside a bioceramic (HA) biocompatible polycaprolactone (PCL) and polyvinylpyrrolidon (PVP) were chosen to prepare electrospun composites of PCL$\mathrm{PVP}-\mathrm{HA}$, for tissue engineering purposes.

HA chemical and crystal structure just resemble to those of bone and tooth minerals, HA is indispensable biomaterial for bone grafting in orthopedics as a filling

\footnotetext{
*e-mail: ymugesahin@arel.edu.tr
}

material [2]. HA can either obtained from commercially or synthesized from bones (i.e. human, animal, fish) as allograph materials. In clinical practice, fresh allografts are rarely used due to the immune response and risk of transmission of diseases. Thus the latter, synthesized HAs are need calcination to get rid of these risks. So, herein as an alternative source of bioceramic, marine shells, were utilized. The synthesis method of this HA, ultrasonication, are effortless and less energetic in comparison to the other methods in the literature such as microwave and hydrothermal methods that need complicated and expensive systems [2] Having biphasic bioceramic formations, these HAs synthesized from natural sources enhance biocompatibility and bioresorbtion [3]

In the proposed study, natural bioceramics were synthesized via ultrasonication method to give mechanical strength and enhanced biological properties to the produced composite whereas, biocompatible and degradable polymers were chosen as the polymeric matrix.

\section{Materials and equipment}

For bioceramic synthesis, Donax trunculus shells were obtained from a gift store and Ortho-phosphoric acid (85\%, reagent purity) were purchased from Merck. Utillized chemicals DMF, chloroform and polymers, PCL and PVP having molecular weights $80,000 \mathrm{~g} / \mathrm{mole}$ and $360,000 \mathrm{~g} / \mathrm{mole}$, were purchased from Sigma-Aldrich and used as they received. 
Donax trunculus shells were cleaned, grinded and sieved under the obtained powder $100 \mu \mathrm{m}$ (Fig. 1). The calcium oxide content of the of the sea shells was determined by thermogravimetric analysis (TGA). Subsequently, shell powder added to $50 \mathrm{ml}$ of distilled water and titrated with orthophosphoric acid at $80^{\circ} \mathrm{C}$ to adjust the $\mathrm{Ca} / \mathrm{P}$ mole ratio as 1.67 for $\mathrm{HA}$ production. After filtration and drying, powders were sintered at $850^{\circ} \mathrm{C}$, to convert into final bioceramic phases.
PCL dissolved in a solvent mixture of chloroform/DMF (60:40) and PVP in DMF in an ultrasonic bath. Three different concentrations $(1 \%, 5 \%$ and $8 \%)$ of $\mathrm{HA}$ were added to the obtained PCL-PVP solution and homojenized at $45^{\circ} \mathrm{C}$ for $1 \mathrm{~h}$ in an ultrasonic bath. Subsequently, the prepared solution was purged to the electrospinning system to obtain the electrospun mats. Structural (XRD, FTIR), morphological (FEGSEM), biological (in-vitro tests) and mechanical (tensile testing) characterizations were conducted for these nanobiomaterials.

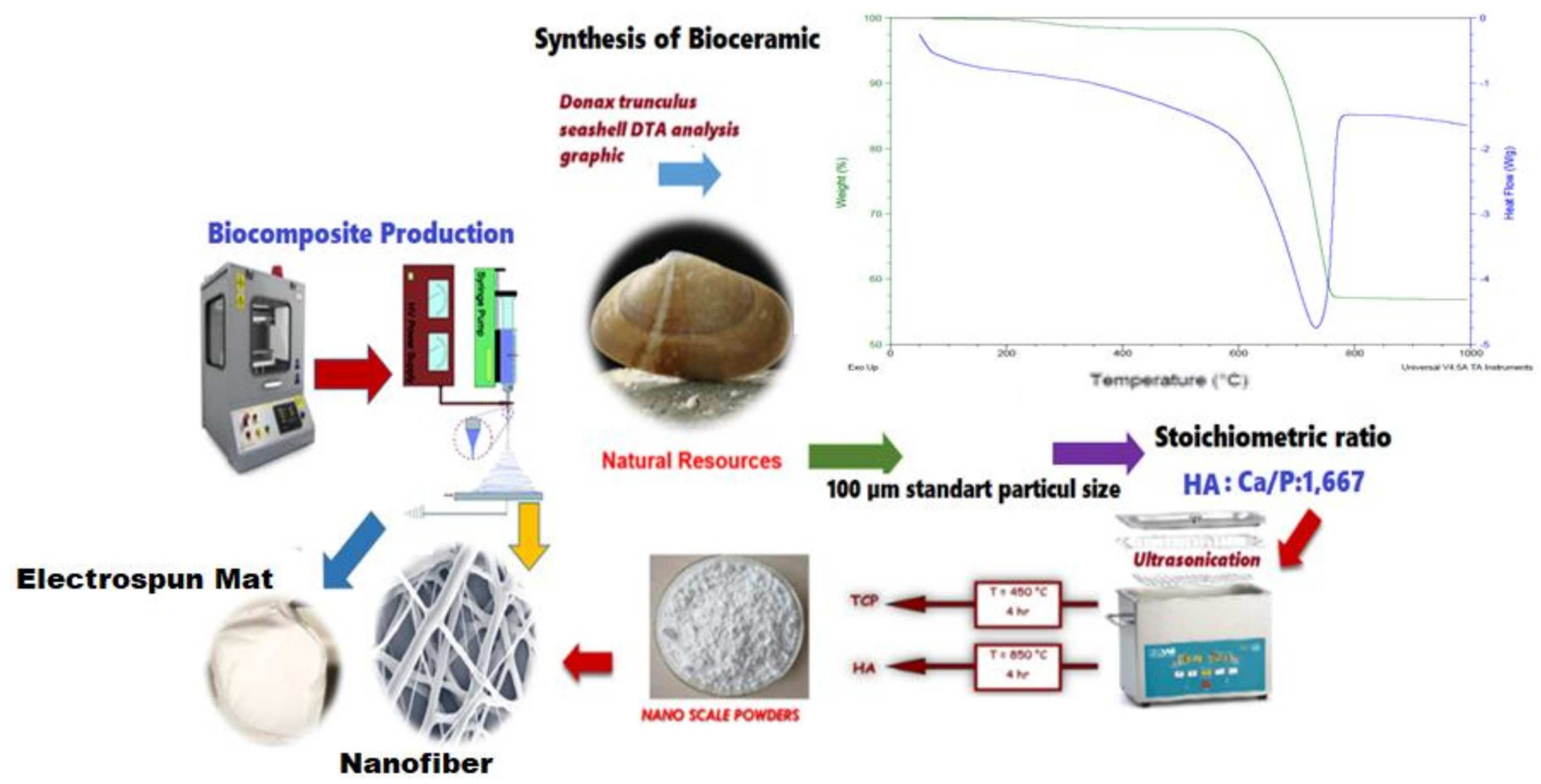

Fig. 1. Bioceramic synthesis and nanobiocomposite mat production processes.

\section{Results and discussion}

\subsection{Structural characteriazations of bioceramic and biocomposites}

In the light of XRD analysis of bioceramic powders, biphasic structures, $97.3 \% \mathrm{HA}$ and $2.7 \% \alpha$-TCP, were obtained (Fig. 2.). Among the most important properties of HA, its perfect biocompatibility can be pronounced. HA chemically bonds to hard tissue and within 4-8 weeks newly formed osteoblast cells accumulates. The osteoconductive properties of HA also allow the implants to firmly adhere to the bone [3]. In addition $\alpha$-TCP can dissolve and gradually degrade in the body. It also supports the formation of new bone by releasing calcium and phosphate ions [4]. The FTIR spectrum of the nanofiber mats were shown in Fig. 3. The bands observed in frequency range of $2945-2866 \mathrm{~cm}^{-1}$ are due to $\mathrm{CH}_{2}$ stretching for PVP and PCL. At $1164 \mathrm{~cm}^{-1}, 1239 \mathrm{~cm}^{-1}$ and $1294 \mathrm{~cm}^{-1}$ symmetrical $\mathrm{C}-\mathrm{O}-\mathrm{C}$ stretching bands were observed for the main polymeric matris, PCL of the composite. The intense peak at $1721 \mathrm{~cm}^{-1}$ is the characteristic carbonyl $(\mathrm{C}=\mathrm{O})$ stretching band of PCL component [5].

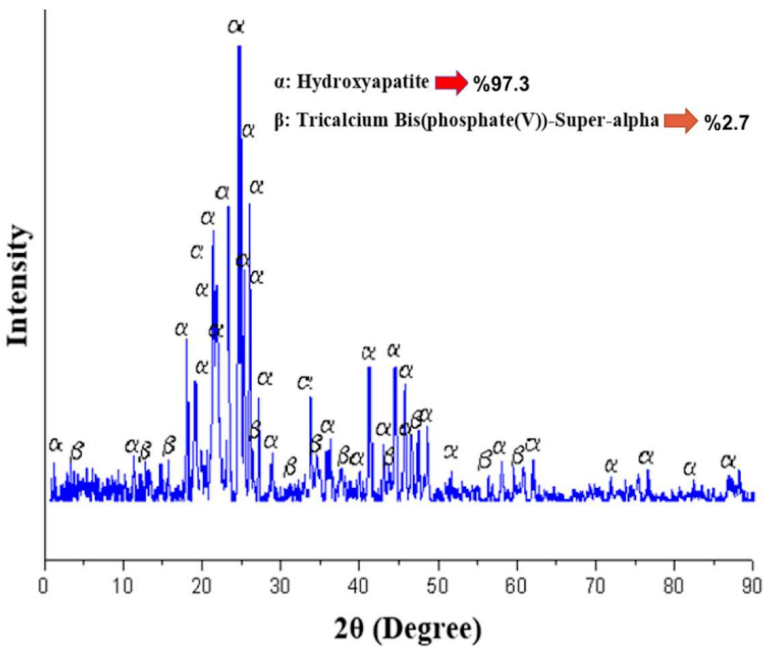

Fig. 2. XRD patterns of the sample sintered at $850^{\circ} \mathrm{C}$.

On the other hand, carbonyl stretching of PVP was observed at $1682 \mathrm{~cm}^{-1}$. Moreover the broad band at $3420 \mathrm{~cm}^{-1}$ is indicative of $\mathrm{O}-\mathrm{H}$ stretching for PVP. 


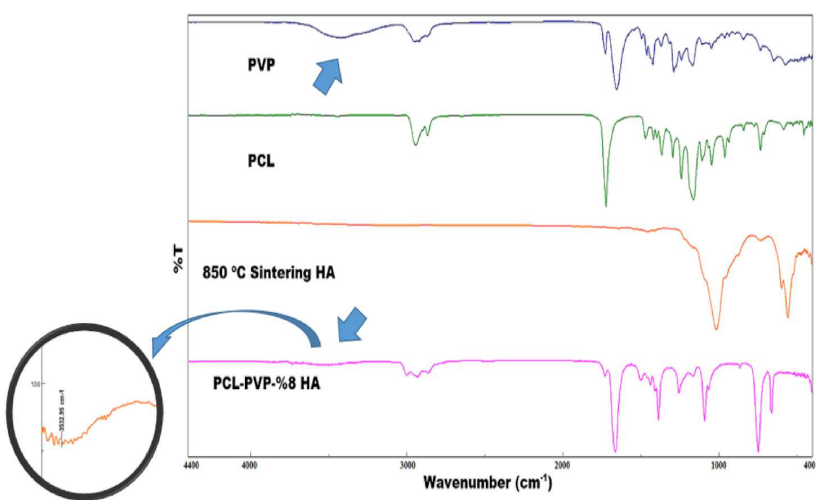

Fig. 3. FTIR spectra of HA, PVP, PCL and nanofiber mats.

The $\mathrm{PO}_{4}^{-3}$ groups of HA gave absorption bands between $1000-1100 \mathrm{~cm}^{-1}$. The additional bands observed at a frequency range of $650-750 \mathrm{~cm}^{-1}$ comes from $\mathrm{HPO}_{4}^{-2}$ group of HA. The $\mathrm{CO}_{3}^{-2}$ group of HA on the other hand, showed a weak peak at a frequency of $872 \mathrm{~cm}^{-1}$ and strong bands between 1430-1530 $\mathrm{cm}^{-1}$ [2]. For PCL-PVP-8\%HA biocomposite, functional group bands overlaped and were in agreement with the polymeric composition. Moreover, different from the polymeric content the obeserved $\mathrm{HPO}_{4}^{-2}$ stretching bands between $650-750 \mathrm{~cm}^{-1}$ were indicative of HA content in the composite.

\subsection{Morphology and size analysis of bioceramic and biocomposites}

Morphology of the samples were examined by FEGSEM, and different allotropic structures were determined for the synthesized bioceramic powder (Figure $4 \mathrm{a}$ and $4 \mathrm{~b}$ ). For electrospun mats (Fig. 4c-h.), nanofiber structures were observed and their mean diameters were calculated by using Image J (2011) software. Average particle diameter of the synthesized bioceramic was $153 \mathrm{~nm}$ whereas, the mean diameters of the fibers for electrospun mats were in the range of 40-220 nm. As HA concentration increases, bioceramic powders coat the polymer fibers homogeneously, resulting in a stronger and finer nanofiber structures [5]. The synthesized natural HA powders in this study, distributes homogeneously on the fibers. The finest fiber diameter were obtained in the PCL/PVP $/ 8 \%$ HA composite (Fig. 4h).
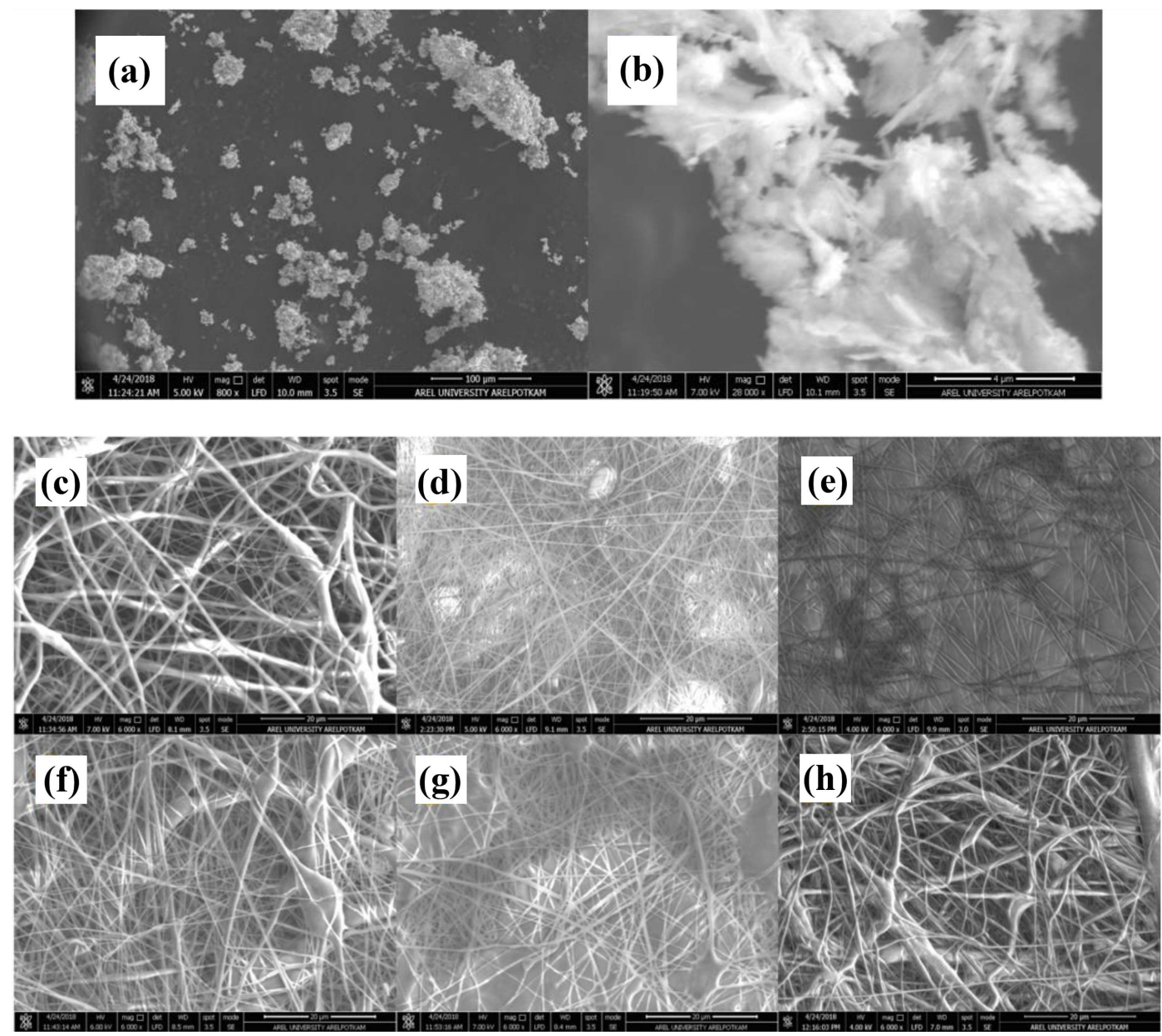

Fig. 4. Microstructure of bioceramic at (a) $\times 800$ (b) $\times 3000$ and nanofiber mats at $\times 6000$ (c) PCL, (d) PVP, (e) PCL-PVP, (f) PCL-PVP-1\%HA, (g) PCL-PVP-5\%HA, (h) PCL-PVP-8\%HA. 


\subsection{Cell viability and mechanical of bioceramic and biocomposites}

Table I summarizes the in vitro tests of the nanobiocomposites. For all nanocomposite mats, live cells, attached to the surface and stained with DAPI (4',6-diamidino-2-phenylindole) were reported. Cell viability of the electrospun mats were found above $94 \%$. By increasing the bioceramic content cell viability percentages were enhanced. As it is revealed below the best cell viability value was observed for the composites containing $8 \%$ of HA.

TABLE I

Cell viability values of biocomposites.

\begin{tabular}{c|c|c|l|c|c}
\hline \hline \multirow{2}{*}{ Sample } & $\begin{array}{c}\text { Living } \\
\text { cells } \\
{[\%]}\end{array}$ & $\begin{array}{c}\text { Dead } \\
\text { cells } \\
{[\%]}\end{array}$ & Sample & $\begin{array}{c}\text { Living } \\
\text { cells } \\
{[\%]}\end{array}$ & $\begin{array}{c}\text { Dead } \\
\text { cells } \\
{[\%]}\end{array}$ \\
\hline control & 97 & 3 & & & \\
average value & 96.0 & 4.0 & & & \\
PCL & 93 & 7 & PCL-PVP-1\%HA & 97 & 3 \\
average value & 94.5 & 5.5 & average value & 95.5 & 4.5 \\
PVP & 94 & 6 & PCL-PVP-\%5HA & 98 & 2 \\
average value & 95.5 & 4.5 & average value & 96.0 & 4.0 \\
PCL-PVP & 94 & 6 & PCL-PVP-\%8HA & 98 & 2 \\
average value & 95.5 & 4.5 & average value & 96.5 & 3.5
\end{tabular}

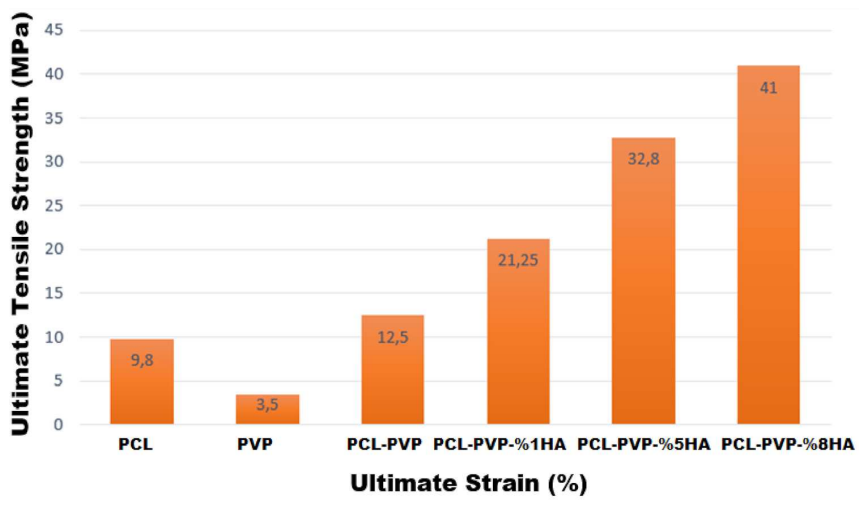

Fig. 5. Tensile test results of nanobiocomposites.

Mechanical properties of the electrospun nanofiber were identified according to ASTM E4 standards and given in Fig. 5. The highest ultimate tensile strength value was obtained as $41.0 \mathrm{MPa}$. Being consistent with morphological investigations, mechanical properties of the composites enhanced as HA content increased. Different electrospun nanobiocomposites are presented in the literature, whereas, the mechanical results obtained in the present work are one of the highest tensile strength among all [5].

\section{Conclusion}

Nanosized natural HA powders were synthesized by ultrasonication method and characterized. In order to overcome the weak mechanical properties of the polymeric matris, a biocompatible ceramic additive was utilized as reinforcer and the mechanical properties of the nanobiocomposites were improved. Since the synthesized natural HA bioceramic powders coated the polymer fibers homogeneously, reinforcing effect was observed and thinner nanofiber mats were obtained. As a consequence, cell adhesion to the scaffold surface was increased and the mechanical properties were improved. Ascending the amount of HA in PCL-PVP nanofiber composite materials, biocompatibility and mechanical strength of biomaterials have been found to increased. The finest fibers were acquired for the PCL-PVP- $8 \%$ HA composite. These nanocomposites, which are produced by using nanotechnology from natural hydroxyapatites, can be ideal materials for tissue engineering due to their biological and mechanical superiority.

\section{Acknowledgments}

I would like to thank to Polymer Technologies and Composite Application and Research Center (ArelPOTKAM) for material characterizations.

\section{References}

[1] F. Anjum, N.A. Agabalyan, H.D. Sparks, N.L. Rosin, M.S. Kallos, J. Biernaskie, Sci. Rep. 7, 10291 (2017).

[2] O. Gunduz, Y.M. Sahin, S. Agathopoulos, B. BenNissan, F.N. Oktar, J. Nanomater. 1, 382861 (2014).

[3] J. Enax, E. Matthias, Oral Health Prev. Dent. 16, 7 (2018).

[4] J.L. Dávila, M.S. Freitas, P.I. Neto, Z.C. Silveira, J.V.L. Silva, M.A. d'Ávila, J. Appl. Polym. Sci. $\mathbf{1 3 3}$ 43031 (2016).

[5] Y.J. Lee, A. Elosegui-Artola, K.H.T. Le, G.M. Kim, Cel. Mol. Bioeng. 6, 482 (2013). 